\title{
Molecular mechanism of epigallocatechin-3-gallate in human esophageal squamous cell carcinoma in vitro and in vivo
}

\author{
LIFENG LIU $^{1 *}$, LEI HOU ${ }^{2 *}$, SHANZHI GU ${ }^{3}$, XIAOXIAO ZUO ${ }^{4}$, DU MENG ${ }^{4}$, \\ MINNA LUO ${ }^{4}$, XIAOJIN ZHANG ${ }^{4}$, SHANGKE HUANG $^{4}$ and XINHAN ZHAO ${ }^{4}$ \\ ${ }^{1}$ Department of Otolaryngology-Head and Neck Surgery, The First Affiliated Hospital of Medical School of Xi'an \\ Jiaotong University, Xi'an, Shaanxi 710061; ${ }^{2}$ Department of Medical Oncology, Shaanxi Provincial People's Hospital, \\ Xi'an, Shaanxi 710068; ${ }^{3}$ Department of Forensic Medicine, Medical School of Xi'an Jiaotong University; \\ ${ }^{4}$ Department of Medical Oncology, The First Affiliated Hospital of Medical School \\ of Xi'an Jiaotong University, Xi'an, Shaanxi 710061, P.R. China
}

Received June 24, 2014; Accepted September 22, 2014

DOI: $10.3892 /$ or.2014.3555

\begin{abstract}
Epigallocatechin-3-gallate (EGCG), the major polyphenol of green tea, has been shown to inhibit proliferation in various types of tumors. However, few studies concerning the role and mechanism of EGCG in esophageal squamous cell carcinoma are available. Therefore, the antitumor mechanism of EGCG needs to be investigated. The present study aimed to examine the antitumor effect of EGCG on the human esophageal squamous cell carcinoma cell lines, Eca-109 and Te-1, in vitro and in vivo. Cell viability was assessed using the MTT assay and tumor formation and growth in murine xenograft models with or without EGCG treatment. Cell cycle analysis and levels of reactive oxygen species (ROS) were detected using flow cytometry. Apoptosis was measured by Annexin/propidium iodide staining. Caspase-3 cleavage and vascular endothelial growth factor (VEGF) expression were detected using western blot analysis and immunohistochemistry in tumor cell lines and tumor xenografts, respectively. The results showed that EGCG inhibited proliferation in the Eca-109 and Te-1 cells in a time- and dose-dependent manner. Tumor cells were arrested in the $G_{1}$ phase and apoptosis was accompanied by ROS production and caspase-3 cleavage. In a mouse model, EGCG significantly inhibited the growth of Eca-109 tumors by
\end{abstract}

Correspondence to: Professor Xinhan Zhao, Department of Medical Oncology, The First Affiliated Hospital of Medical School of Xi'an Jiaotong University, 227 Yanta West Road, Xi'an, Shaanxi 710061, P.R. China

E-mail: zhaoxinhanprof@163.com

Professor Shanzhi Gu, Department of Forensic Medicine, Medical School of Xi'an Jiaotong University, 76 Yanta West Road, Xi'an, Shaanxi 710061, P.R. China

E-mail: gushanzhi@mail.xjtu.edu.cn

*Contributed equally

Key words: EGCG, ESCC, apoptosis, ROS, caspase-3, VEGF increasing the expression of cleaved-caspase-3 and decreasing VEGF protein levels. Taken together, the results suggest that EGCG inhibits proliferation and induces apoptosis through ROS production, caspase-3 activation, and a decrease in VEGF expression in vitro and in vivo. Furthermore, EGCG may have future clinical applications for novel approaches to treat esophageal squamous cell carcinoma.

\section{Introduction}

Esophageal cancer (EC) is a malignant tumor of the esophagus. There are two main subtypes of EC: esophageal adenocarcinoma $(E A C)$ and esophageal squamous cell carcinoma (ESCC). EAC is common in Western countries, while ESCC is common in East Asia, particularly in China (1). Over 600,000 new cases are diagnosed annually worldwide (2). ESCC is often locally advanced by the time patients are preovided with medical attention and since surgery is the only curative treatment option, patient survival is closely associated with the stage of disease (3-6). Despite improvements in diagnosis and treatment, the 5-year survival rate for patients with advanced and metastatic EC remains $<20 \%$ after surgery (1).

Epigallocatechin-3-gallate (EGCG), a major polyphenolic constituent of green tea, has been shown to inhibit cancer growth and induce apoptosis in hepatocellular carcinoma, breast, and head and neck cancers (7-9). EGCG has been suggested to contribute to the effect of anticancer drugs by increasing cell cycle arrest, initiating apoptosis and downregulating the pro-angiogenic molecule, vascular endothelial growth factor receptor-2 (VEGFR-2) (10). Results of recent studies have shown that EGCG has different effects on the production of reactive oxygen species (ROS) for its antioxidant and pro-oxidant activities $(10,11)$. Caspases are key regulators of apoptosis, with caspase-3 acting as an effector (12). Additionally, caspase-3 was found to play a role in EGCGinduced apoptosis for cholangiocarcinoma and laryngeal epidermoid carcinoma $(13,14)$. In addition, downregulation of the pro-angiogenic factor, VEGF has been documented to inhibit lung cancer growth (15). Few reports are available on the effect of EGCG on esophageal squamous cell carcinoma. 
Therefore, we examined the mechanism of EGCG by studying apoptosis, ROS generation, cleaved caspase-3 and VEGF expression in Eca-109 and Te-1 cell lines.

\section{Materials and methods}

Cell culture and reagents. 3-(4,5-Dimethylthiazol-2-yl)-2,5diphenyltetrazolium bromide (MTT) was purchased from Sigma (St. Louis, MO, USA). Dulbecco's modified Eagle's medium(DMEM) and fetal bovine serum(FBS) were purchased from HyClone (Logan, UT, USA). Antibodies against cleaved caspase-3 and VEGF were purchased from Cell Signaling Technology (Danvers, MA, USA) and the Proteintech Group (Chicago, IL, USA), respectively. Horseradish peroxidaselabeled secondary antibodies were purchased from Sigma.

The H9c2 rat cardiomyocyte cell line and Eca-109 and Te-1 human esophageal squamous cell carcinoma cell lines were purchased from the Cell Bank of the Chinese Academy of Sciences (Shanghai, China). Human foreskin fibroblast cells were a kind gift from Dr Ming Zhang (Department of Cardiovascular Medicine, The Second Affiliated Hospital of Xi'an Jiaotong University). Cell lines were cultured in DMEM-H medium supplemented with $10 \%$ FBS and $1 \%$ (100 U/ml) penicillin/streptomycin (Sigma-Aldrich Co.). The culture medium was changed every two days. Upon reaching $80-90 \%$ confluency, the cells were passaged and tested for logarithmic growth.

MTT assay. To assess the effect of EGCG on cell growth, H9c2 rat cardiomyocytes, HFF, Eca-109 and Te-1 cells $\left(1 \times 10^{4}\right.$ cells/well) were inoculated into a 96-well microtiter plate and cultured for $24 \mathrm{~h}$. The cells were treated with various concentrations of EGCG $(25,50,100,200$ and $400 \mu \mathrm{M})$ or vehicle control (DMSO). After 24 and $48 \mathrm{~h}$ of incubation, $20 \mu \mathrm{l} 5 \mathrm{mg} / \mathrm{ml}$ MTT was added to each well for $4 \mathrm{~h}$ of incubation. The absorbance was measured at $490 \mathrm{~nm}$ using a multi-channel microplate reader (BD Biosciences, San Jose, CA, USA). The inhibition rate of cell growth was determined using the formula: (OD value for control group - OD value of experimental group)/OD value of the control group x $100 \%$. The $\mathrm{IC}_{50}$ value for EGCG was determined using GraphPad Prism software (version 5.0).

Cell cycle analysis. Cell cycle distribution was analyzed by flow cytometry using propidium iodide (PI) DNA staining as previously described with modifications (16). Briefly, Eca-109 and Te-1 were treated with 256 and $162 \mu \mathrm{M}$ EGCG $\left(\mathrm{IC}_{50}\right)$ value, respectively, for $24 \mathrm{~h}$ in serum-free DMEM. The cells were collected, washed with phosphate-buffered saline (PBS), centrifuged at 1,000 rpm for $5 \mathrm{~min}$, and fixed in $5 \mathrm{ml}$ of $70 \%$ ice-cold ethanol for $24 \mathrm{~h}$ at $4^{\circ} \mathrm{C}$. After washing, the pellets were resuspended in $2.5 \mu \mathrm{l}$ of ribonuclease A solution (final concentration of $50 \mu \mathrm{g} / \mathrm{ml}$ ) and incubated at $37^{\circ} \mathrm{C}$ for $30 \mathrm{~min}$. Cell suspensions received $5 \mu \mathrm{l}$ PI (final concentration of $50 \mu \mathrm{g} /$ $\mathrm{ml}$ ), were protected from light, and incubated at room temperature for $30 \mathrm{~min}$. Flow cytometric analysis of DNA content was carried out using the FACSCalibur system (BD Biosciences).

Annexin V-FITC/PI staining. To analyze the effect of EGCG on apoptotic cell death, Annexin V-FITC/PI staining was performed as previously described and modified (17). Briefly, the cells were treated with EGCG $\left(\mathrm{IC}_{50}\right)$ value for $24 \mathrm{~h}$ in serum-free DMEM and gently washed three times in PBS. The cells were collected, centrifuged at 1,000 rpm for $5 \mathrm{~min}$, and washed with PBS. A cell density of $5 \times 10^{5}$ was resuspended in $500 \mu 11 \mathrm{X}$ binding buffer and stained with $5 \mu \mathrm{l}$ Annexin V-FITC and $10 \mu \mathrm{l}$ PI staining solution. The cell suspension was covered and incubated for $15 \mathrm{~min}$ at room temperature. The experiments were performed three times and analyzed using the FACSCalibur system.

ROS production. Eca-109 and Te-1 cells $\left(2 \times 10^{5}\right.$ cells/well $)$ were seeded into a 6 -well plate and treated with EGCG $\left(\mathrm{IC}_{50}\right)$ value in serum-free DMEM for $24 \mathrm{~h}$. After $24 \mathrm{~h}$, cells $\left(5 \times 10^{5}\right.$ cells $\left./ \mathrm{ml}\right)$ were resuspended in serum-free DMEM and labeled with $1 \mathrm{ml}$ DCFH-DA dye $(1 \mu \mathrm{g} / \mu \mathrm{l})$ for $20 \mathrm{~min}$ with continuous agitation. FACS (BD Co.) analysis detected the oxidative burst (hydrogen peroxide).

Western blotting. Immunoblot analysis was performed as described and modified (18). Eca-109 and Te-1 cells $\left(1 \times 10^{5}\right.$ cells $\left./ \mathrm{ml}\right)$ were treated with or without EGCG for $24 \mathrm{~h}$. Cell lysates ( $20 \mu \mathrm{g}$ protein) were separated by SDS-PAGE, transferred to polyvinylidene difluoride membranes (Millipore, Bedford, MA, USA), and blocked. The following primary antibodies were applied overnight at $4^{\circ} \mathrm{C}$ : cleaved caspase-3 $(1: 1,000)$ and VEGF $(1: 1,000)$. Anti-rabbit secondary antibodies $(1: 5,000)$ were incubated for $2 \mathrm{~h}$ at room temperature.

Mice and xenograft models. Male BALB/c (nu-nu) athymic nude mice (4-5 weeks of age) were purchased from the Shanghai Silaike Laboratory Animal Company, Ltd. (Shanghai, China). The mice were maintained at Xi'an Jiatong University in compliance with the Institutional Animal Care and Use Committee (IACUC) regulations. For subcutaneous implantation, Eca-109 cells $\left(2 \times 10^{6}\right)$ were suspended in serum-free DMEM mixed with equal parts of Matrigel (BD Biosciences, San Diego, CA, USA) and subcutaneously injected into the flank of nude mice $(\mathrm{n}=30)$. Engrafted mice received weekly visual inspections and palpitations until tumors reached $50 \mathrm{~mm}^{3}$ in size. Xenografts were randomly divided into the EGCG- and PBS-treated groups. Experimental mice received intraperitoneal injections of EGCG $(10 \mathrm{mg} / \mathrm{kg})$ once a day for two weeks while control mice were injected with $200 \mu \mathrm{l}$ PBS (19). The tumor volume was measured using a caliper and calculated using the formula: (width in $\mathrm{mm})^{2} \mathrm{x}$ (length in $\mathrm{mm}$ )/2. The mice were sacrificed by cervical dislocation. Subcutaneous tumors, liver, lung and kidney tissues were harvested, fixed in $4 \%$ paraformaldehyde, and embedded in paraffin. Paraffin sections $(4-\mu \mathrm{m})$ were histopathologically evaluated.

Immunohistochemical and histological analyses. Immunohistochemistry was performed as described and modified (20). Antigen retrieval of serial sections was conducted with a 3-min microwave treatment in $10 \mathrm{mM}$ sodium citrate buffer ( $\mathrm{pH}$ 6.0). The sections were incubated with primary antibodies against cleaved caspase-3 (1:100) and VEGF (1:100). The sections were then incubated with biotinylated anti-rabbit antibodies $(1: 100)$ at $37^{\circ} \mathrm{C}$ for $30 \mathrm{~min}$, followed by a 30 -min exposure to streptavidin-horseradish peroxidase (1:200), and counterstained with 

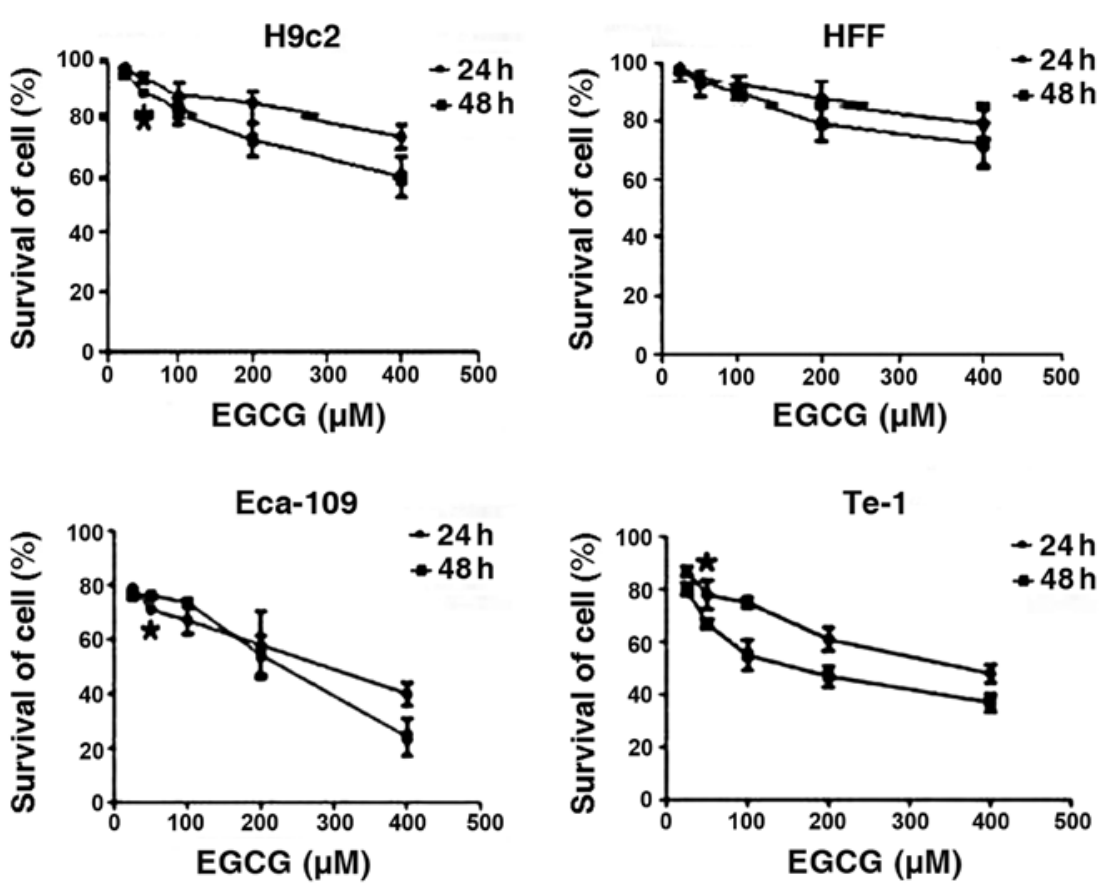

Figure 1. EGCG inhibits cell growth. Rat cardiomyocytes, human foreskin fibroblasts (HFF), Eca-109 and Te-1 cells were treated with increasing doses of EGCG $(25,50,100,200$ and $400 \mu \mathrm{M})$ for 24 and $48 \mathrm{~h}$. The experiments were performed three times with five replicates in each, "p $<0.05$. EGCG, epigallocatechin-3-gallate.

hematoxylin. Optical density was analyzed with Image-Pro Plus software (version 6.0). Hematoxylin and eosin (H\&E) staining was used to evaluate normal liver, lung and kidney tissues.

Statistical analysis. A one-way ANOVA and Dunnett's t-test were used to analyze the significant differences between treatment groups. Statistical analysis was performed using SPSS (version 17.0). The results are expressed as means \pm SD. $\mathrm{P}<0.05$ was considered to indicate a statistically significant result.

\section{Results}

EGCG inhibits cell growth in a time-and dose-dependent manner. In order to ascertain the capability of EGCG to inhibit cell growth, we studied the effect of EGCG treatment on Eca-109 and Te-1 cells compared with normal rat cardiomyocytes and HFF cells. The results revealed that, 24 and $48 \mathrm{~h}$ treatment with EGCG selectively inhibited the growth of the Eca-109 and Te-1 cells in a time- and dose-dependent manner when the concentration was $>25 \mu \mathrm{M}$. For Te-1 cells EGCG inhibition of cell growth was more effective at $48 \mathrm{~h}$ posttreatment than that at $24 \mathrm{~h}$ when the concentration remained unchanged. For Eca-109 cells it appeared that the effect of EGCG was more effective at $48 \mathrm{~h}$ post-treatment when the concentration was $>200 \mu \mathrm{M}$. The estimated $\mathrm{IC}_{50}$ value for rat H9c2 cardiomyocytes, HFF, Eca-109 and Te-1 cell lines was 995, 1,584, 256 and $162 \mu \mathrm{M}$, respectively (Fig. 1).

EGCG arrests Eca-109 and Te-1 cells in the $G_{1}$ phase. We analyzed the DNA content of Eca-109 and Te-1 cells treated with the EGCG $\mathrm{IC}_{50}$ value for $24 \mathrm{~h}$. FACS analysis showed a significant increase in the percentage of Eca-109 (from
$44.49 \pm 3.32$ to $58.45 \pm 7.78 \%$ ) and Te-1 cells (from $39.69 \pm 4.23$ to $53.66 \pm 3.87 \%$ ) in the $G_{1}$ phase compared to control-treated cells. Furthermore, there was a decrease in the percentage of Eca-109 cells (from $27.29 \pm 4.21$ to $12.73 \pm 2.89 \%$ ) and Te-1 cells (from $27 \pm 2.67$ to $11.14 \pm 3.06 \%$ ) in the $\mathrm{G}_{2} / \mathrm{M}$ phase. These results demonstrated that EGCG can arrest Eca-109 and Te-1 cells at the $G_{1}$ checkpoint of the cell cycle (Fig. 2).

EGCG induces apoptosis in Eca-109 and Te-1 cells. Annexin V-FITC/PI-labeled cells were treated with EGCG and analyzed by FACS to determine the apoptotic rate. Twenty-four hours of treatment with EGCG significantly increased the percentage of apoptotic Eca-109 cells $(6.13 \pm 1.65$ to $32.23 \pm 7.23 \%)$ and Te-1 cells $(8.22 \pm 2.78$ to $27.7 \pm 9.35 \%)$. The results indicated that EGCG had an obvious effect on inducing apoptosis (Fig. 3).

EGCG induces ROS production in Eca-109 and Te-1 cells. Since we observed that EGCG induces apoptosis, we examined the production of ROS in Eca-109 and Te-1 cells treated with an EGCG $\mathrm{IC}_{50}$ value for $24 \mathrm{~h}$. Using the ROS-sensitive probe, DCFH-DA, we found that the fluorescent intensity was significantly increased in Eca-109 and Te-1 cells compared to the control-treated cells (Fig. 4).

EGCG exhibits differing effects on cleaved caspase-3 and $V E G F$ expression. We assessed the effects of EGCG on the expression of cleaved caspase-3 and VEGF by western blotting. Cleaved caspase-3 levels were significantly increased in Eca-109 and Te-1 cells after 24 h of treatment with the EGCG $\mathrm{IC}_{50}$ value while VEGF expression was decreased significantly (Fig. 5). The results suggested that EGCG can simultaneously increase cleaved caspase-3 and decrease VEGF protein levels. 


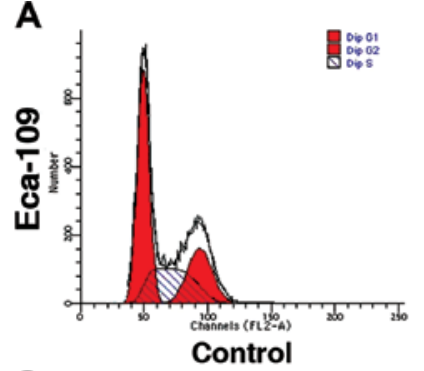

C

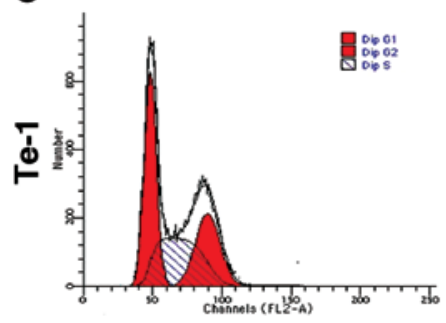

Control

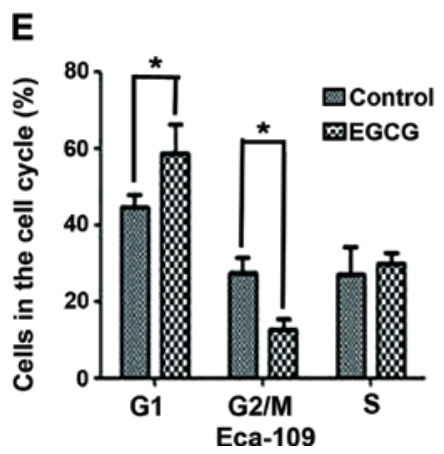

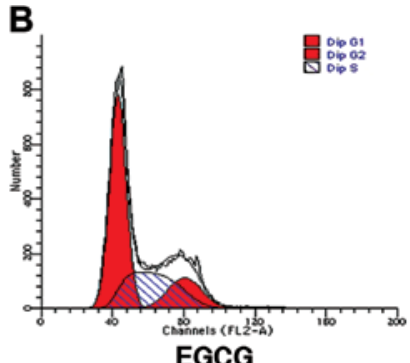

D

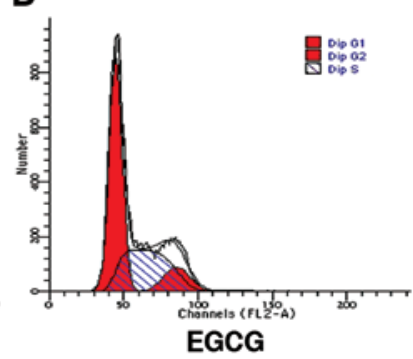

$\mathbf{F}$

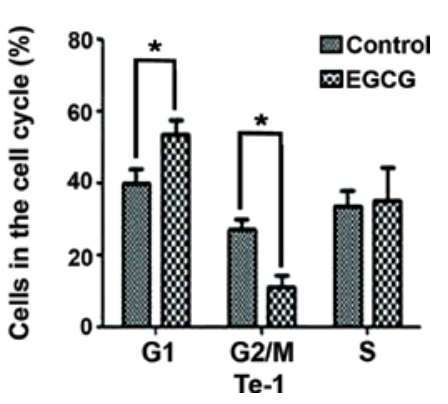

Figure 2. EGCG arrests Eca-109 and Te-1 cells in the $\mathrm{G}_{1}$ phase. (A and C) Eca-109 and Te-1 cells treated with PBS control. (B) Eca-109 and (D) Te-1 cells treated with EGCG for $24 \mathrm{~h}$. (E and F) Graphical representation of the percentage of cells in the $G_{1}, G_{2} / M$ and $S$ phases of the cell cycle. The first peak contains cells with diploid DNA at $\mathrm{G}_{1}$. The cells in the second peak with double PI-fluorescence intensity are tetraploid at $\mathrm{G}_{2}$. The area between the two peaks shows cells in the $\mathrm{S}$ phase. The results suggest that EGCG-treated Eca-109 and Te-1 cells were prominently arrested at the $\mathrm{G}_{1}$ phase of the cell cycle, ${ }^{*} \mathrm{p}<0.05$. Representative histograms are shown. EGCG, epigallocatechin-3-gallate.

EGCG inhibits tumor growth in vivo. To expand the in vitro studies, we tested the effects of EGCG on tumor growth in vivo. Eca-109 EC cells were subcutaneously implanted in the flank of nude mice for two weeks (Fig. 6). Tumor tissues were processed and analyzed by immunohistochemistry for cleaved caspase-3 and VEGF proteins (Fig. 7). Consistent with the in vitro data, we confirmed that EGCG significantly inhibited tumor growth by increasing caspase-3 cleavage and decreasing VEGF protein expression. Furthermore, H\&E staining showed that EGCG was non-toxic to normal tissues (Fig. 8).

\section{Discussion}

In the present study, we have demonstrated that EGCG is able to inhibit the growth of malignant Eca-109 and Te-1 esophageal cancer cells in vitro and in vivo. Eca-109 and Te-1 cells were arrested in the $G_{1}$ phase of the cell cycle and underwent apoptotic cell death following exposure to EGCG. ROS production was increased in Eca-109 and Te-1 cells in vitro. The same effect has been reported in in vitro studies on human

A

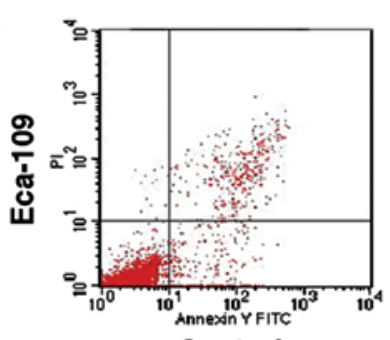

Control

C

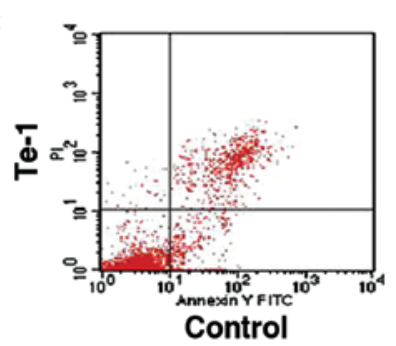

E

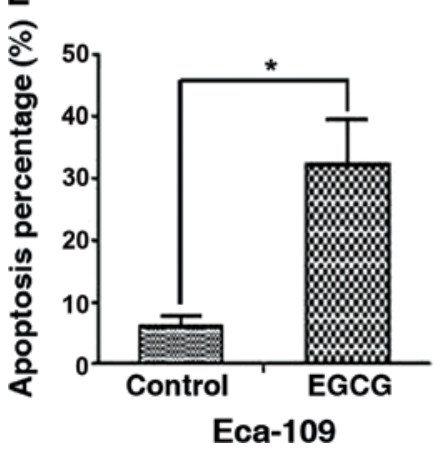

B

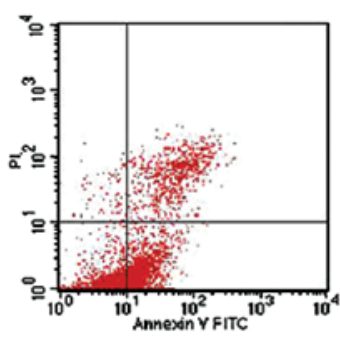

EGCG

D

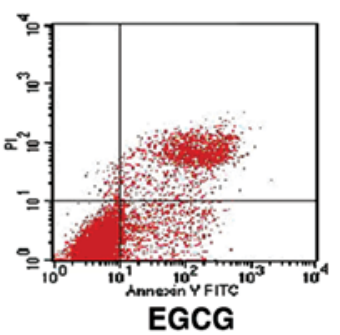

$\mathbf{F}$

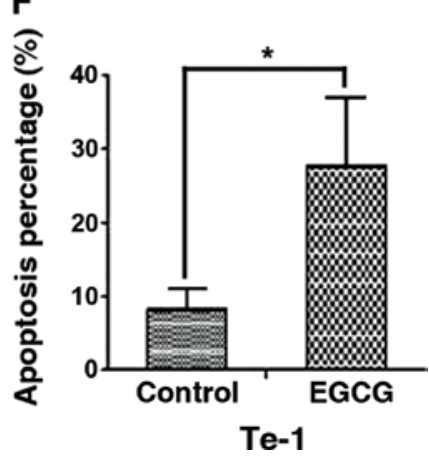

Figure 3. Flow cytometric analysis of apoptosis in human esophageal cancer cells. (A and C) FITC-labeled Eca-109 and Te-1 cells treated with PBS control. (B) Eca-109 and (D) Te-1 cells treated with EGCG for $24 \mathrm{~h}$. (E and F) Graphical representation of the percentage of apoptotic cells. The results indicate that EGCG can significantly induce apoptotic cell death in human Eca-109 and Te-1 cells, ${ }^{*}$ p<0.05. EGCG, epigallocatechin-3-gallate.

cervical cancer and hepatocellular carcinoma $(21,22)$. EGCGtreated xenograft models demonstrated reduced tumor growth, an increased expression of cleaved caspase-3, and decreased VEGF protein levels. The results suggest that EGCG effectively inhibits esophageal squamous cell carcinoma by inducing apoptosis and caspase- 3 expression and suppressing VEGF expression.

Conflicting results have been reported regarding cell cycle arrest by EGCG. Findings of a recent study showed that EGCG induced cell cycle arrest in $\mathrm{G}_{2} / \mathrm{M}$ phase in the epithelioid malignant mesothelioma-derived REN cells (18). Ma et al (23) reported that poorly differentiated AGS gastric cancer cells were arrested at S phase by EGCG. Thakur et al (24) demonstrated that HCT116 colon cancer cells were arrested at $\mathrm{G}_{1}$ phase. In the present study, we observed that Eca-109 and Te-1 cells were arrested at $\mathrm{G}_{1}$ phase.

EGCG acts as an antioxidant (25), and possesses significant pro-oxidant activity (26). Reactive oxygen species (ROS) were reported to be responsible for EGCG-induced apoptosis in mesothelioma (27) and EGCG-induced ROS production in endometrial carcinoma cells (28). However, ROS were not involved in EGCG-induced apoptosis in human laryngeal epidermoid carcinoma (Hep2) cells (14), although EGCG 
A

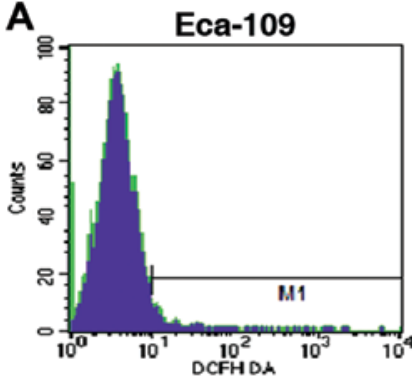

Blank control
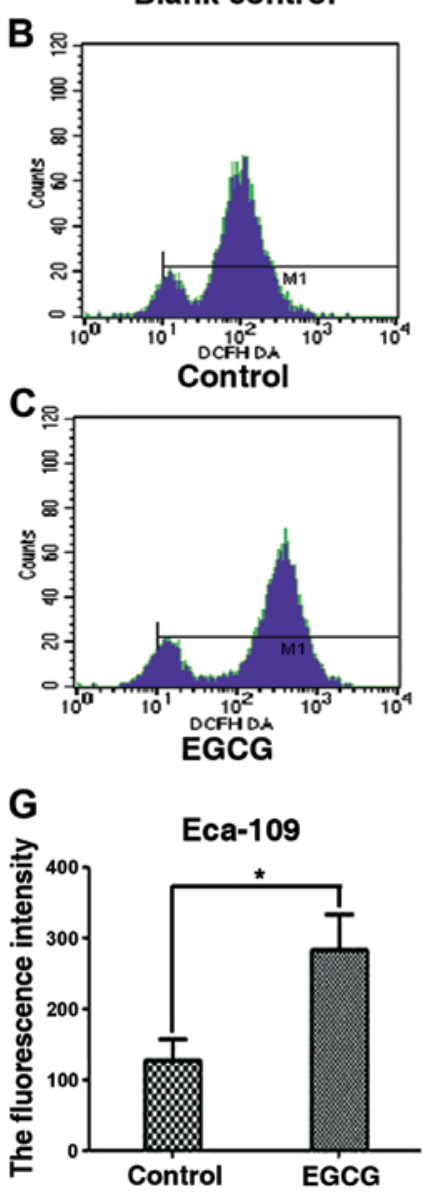

D

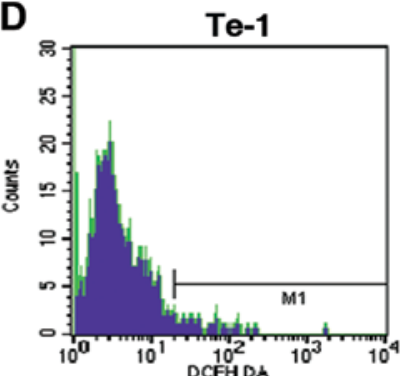

Blank control

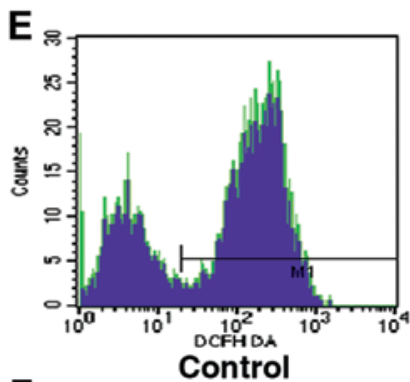

$\mathbf{F}$

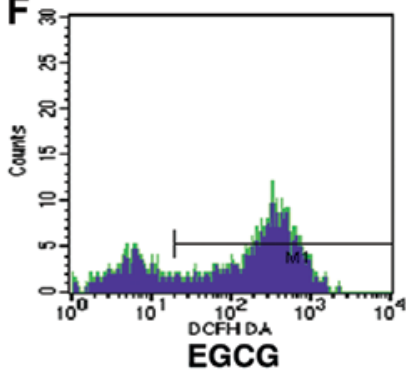

H

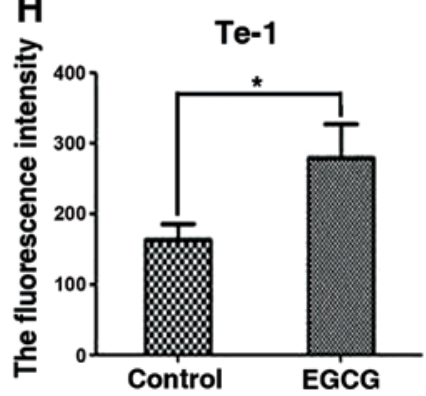

Figure 4. Flow cytometric analysis of ROS production in Eca-109 and Te-1 cells. Reactive oxygen species (ROS) were detected using the FACScan system. (A and B) Blank control and Eca-109 cells treated with PBS control, respectively. (C) Eca-109 cells treated with an EGCG $\mathrm{IC}_{50}$ value for $24 \mathrm{~h}$. (D and E) Blank control and Te-1 cells treated with DMSO control, (F) Te-1 cells treated with an EGCG $\mathrm{IC}_{50}$ value for $24 \mathrm{~h}$. (G and $\mathrm{H}$ ) Graphical representation of the fluorescent intensity of Eca-109 and Te-1 cells, respectively. The experiments were performed three times. The data suggest that EGCG can significantly induce ROS in human Eca-109 and Te-1 cells, ${ }^{*} \mathrm{p}<0.05$. EGCG, epigallocatechin-3-gallate.

reduced deoxynivalenol-induced ROS in HT-29 cells (29). In the present study, ROS were found to contribute to EGCG-induced apoptosis in Eca-109 and Te-1 cells.

In the present study, cleaved caspase-3 expression was increased by EGCG in vitro and in vivo. EGCG was reported to sensitize hepatocellular carcinoma $\mathrm{HepG}_{2}$ cells to apoptosis by increasing caspase- 3 activity (7). Mitochondria play a crucial role in many apoptotic responses (30). Stress signals cause mitochondria to release cytochrome $c$, leading to activated caspase- 3 as the critical executioner of apoptosis (31). In

A
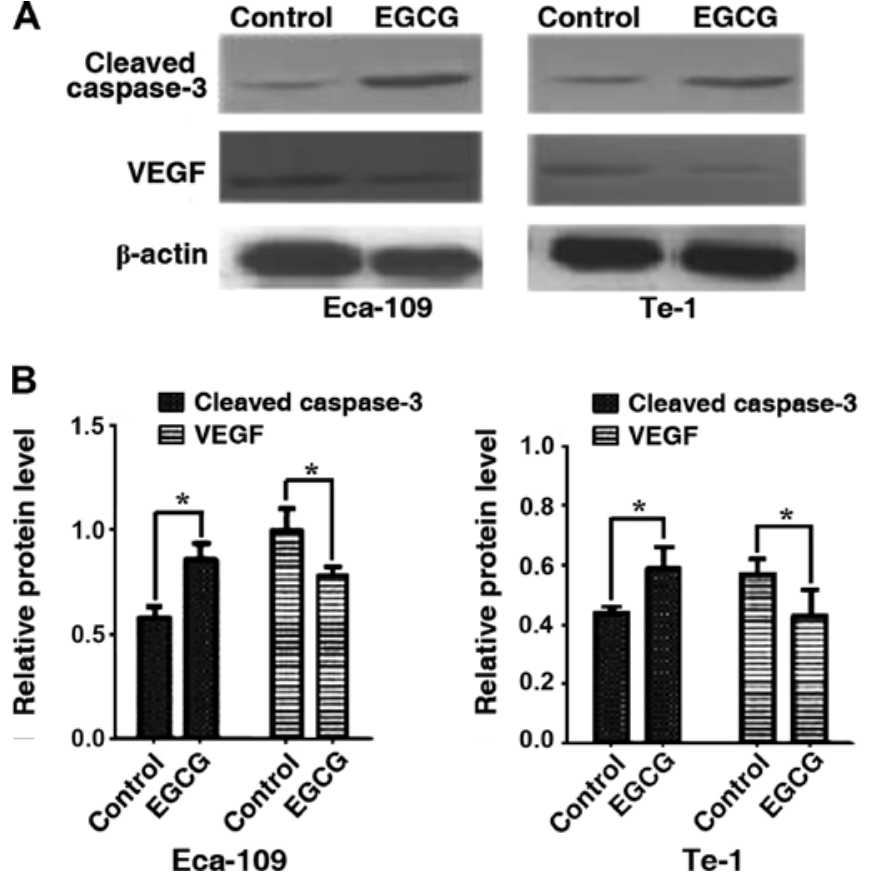

Figure 5. EGCG increases cleaved caspase-3 levels and decreases VEGF expression. (A) Eca-109 and Te-1 cells treated with PBS control or EGCG for $24 \mathrm{~h}$. Western blot analysis was performed using anti-cleaved caspase-3 and VEGF antibodies. $\beta$-actin was used as an internal control. Western blot analyses were repeated three times. (B) Graphical representation of optical densities were compared between EGCG-treated and untreated control cells, " $\mathrm{p}<0.05$. EGCG, epigallocatechin-3-gallate; VEGF, vascular endothelial growth factor.

\section{A}

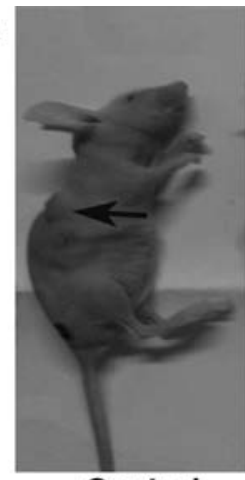

Control

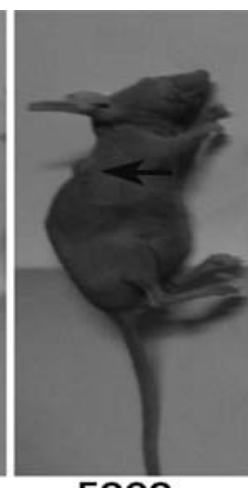

EGCG

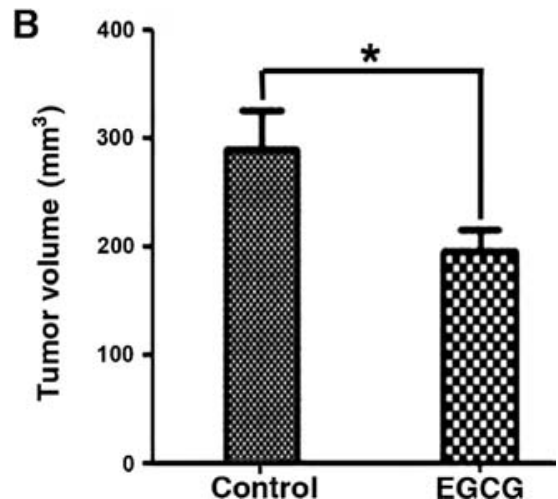

Figure 6. EGCG inhibits Eca-109 tumor formation in a xenograft model (A) Images of mice harboring Eca-109 tumors 14 days after implantation. (B) Graphical representation of tumor volume in EGCG-treated (intraperitoneal injection, $10 \mathrm{mg} / \mathrm{kg} /$ day) control-treated mice. The data show that EGCG can significantly inhibit Eca-109 tumor formation in nude mice, ${ }^{*} \mathrm{p}<0.05$ EGCG, epigallocatechin-3-gallate. 


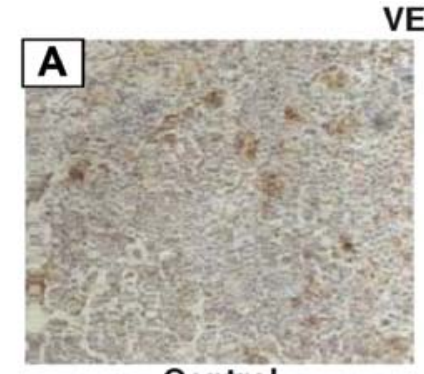

Control

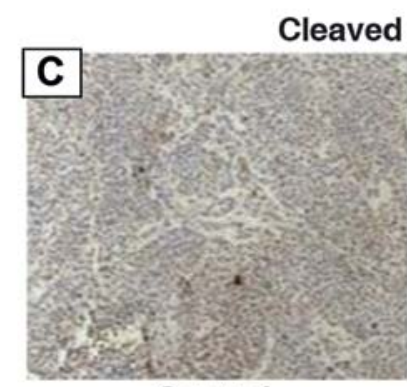

Control

E

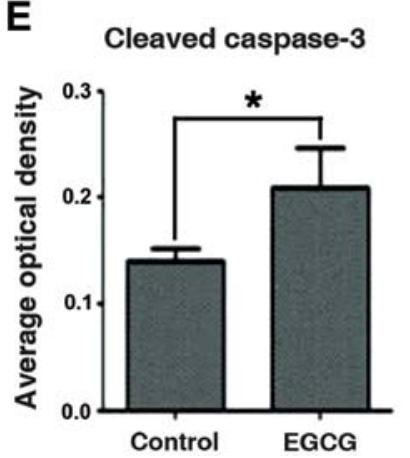

VEGF

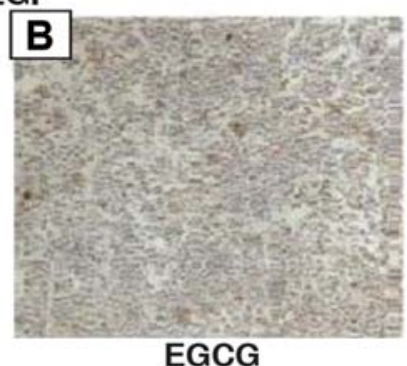

EGCG

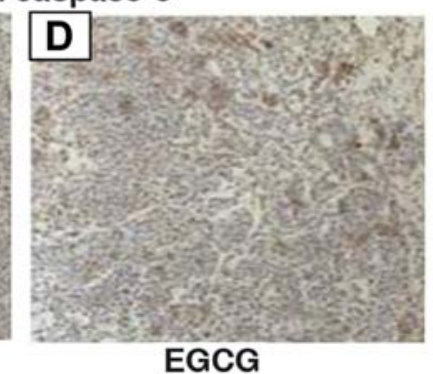

$\mathbf{F}$

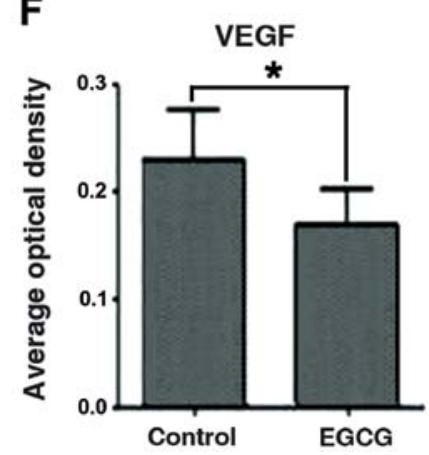

Figure 7. Immunohistochemical analysis of tumor tissue. Eca-109 tumors were harvested from subcutaneously injected mice two weeks after tumor implantation and intraperitoneal injection of EGCG $(10 \mathrm{mg} / \mathrm{kg} / \mathrm{day})$ Tumor sections were processed and stained for (B) cleaved caspase-3, and (D) VEGF. (A and C) The control-treated group is shown. (E and F) Optical densities were determined two weeks after EGCG treatment, ${ }^{*} \mathrm{p}<0.05$. EGCG, epigallocatechin-3-gallate; VEGF, vascular endothelial growth factor.

endometrial carcinoma, high levels of ROS caused oxidative stress and EGCG was demonstrated to induce apoptosis by ROS generation and increase caspase-3 (28). Consistent with the findings of the aforementioned studies, the present study reports that EGCG-induced apoptosis in Eca-109 and Te-1 cell lines by increasing caspase-3 activity and ROS generation. Due to cross signaling of intrinsic and extrinsic apoptotic pathways, more studies are required to elucidate the molecular mechanism involved.

The VEGF signaling pathway has been identified in the angiogenic process $(32,33)$. This process is required for the growth of normal and tumor tissues such as esophageal carcinomas (34). Consistent with in vitro and in vivo results from the present study, EGCG has been shown to inhibit tumor growth by downregulating VEGF expression $(35,36)$.

Although EGCG shows a cytoprotective effect at low concentrations $(10-20 \mu \mathrm{M})(37)$, it has been reported that consumption of green tea-derived supplements at a high dose $(120 \mathrm{mg} / \mathrm{kg})$ can produce toxic effects in rodents (38).
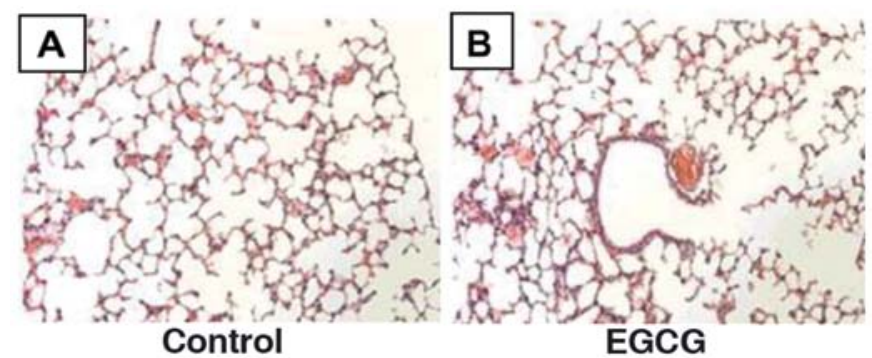

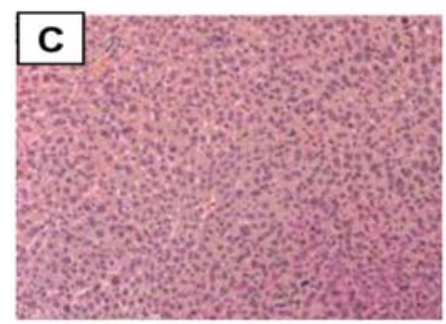

Control

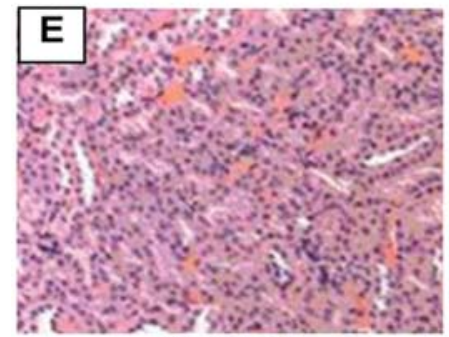

Control

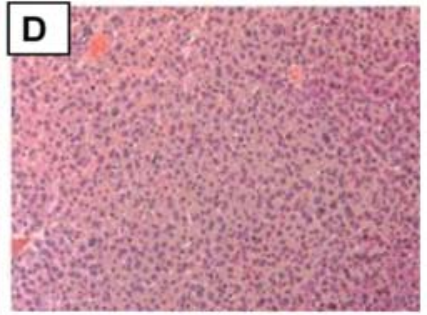

EGCG

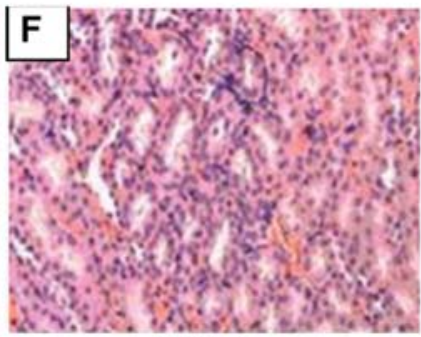

EGCG
Figure 8. Histological evaluation of normal tissues in response to EGCG treatment. Two-week treatment with EGCG showed no toxicity in (B) normal lung, (D) liver and (F) kidney tissues. (A, C and E) Control-treated lung, liver and kidney tissues, respectively, are shown. (A-F) Magnification, x200. EGCG, epigallocatechin-3-gallate.

However, in the present study we found that EGCG showed no obvious toxicity in normal rat cardiomyocytes, human foreskin fibroblast, liver, spleen or kidney tissues of xenograft mice. The results may be due to a selective effect on certain types of cancer (39). Thus, additional investigations into EGCG toxicity are necessary.

In conclusion, our in vitro and in vivo studies confirmed the growth inhibition of human esophageal carcinoma cell lines, Eca-109 and Te-1 in xenograft models. EGCG arrested the growth of cancer cells in the $G_{1}$ phase, induced apoptosis and ROS generation, decreased VEGF levels, and activated caspase-3 without affecting normal tissues.

\section{Acknowledgements}

We would like to thank Professor Chen Huang at Xi'an Jiaotong University for providing the platform to conduct experiments and giving expert advice, and Dr Ming Zhang for sharing the human foreskin fibroblast cell line.

\section{References}

1. Demeester SR: Epidemiology and biology of esophageal cancer. Gastrointest Cancer Res 3 (Suppl 1): S2-S5, 2009.

2. Jemal A, Bray F, Center MM, Ferlay J, Ward E and Forman D: Global cancer statistics. CA Cancer J Clin 61: 69-90, 2011. 
3. Chowdhury FU, Bradley KM and Gleeson FV: The role of ${ }^{18} \mathrm{~F}$-FDG PET/CT in the evaluation of oesophageal carcinoma. Clin Radiol 63: 1297-1309, 2008.

4. Choi J, Kim SG, Kim JS, Jung HC and Song IS: Comparison of endoscopic ultrasonography (EUS), positron emission tomography (PET), and computed tomography (CT) in the preoperative locoregional staging of resectable esophageal cancer. Surg Endosc 24: 1380-1386, 2010.

5. Salahudeen HM, Balan A, Naik K, Mirsadraee S and Scarsbrook AF: Impact of the introduction of integrated PET-CT into the preoperative staging pathway of patients with potentially operable oesophageal carcinoma. Clin Radiol 63: 765-773, 2008

6. Noble F, Bailey D; SWCIS Upper Gastrointestinal Tumour Panel, Tung K and Byrne JP: Impact of integrated PET/CT in the staging of oesophageal cancer: a UK population-based cohort study. Clin Radiol 64: 699-705, 2009.

7. Abou El Naga RN, Azab SS, El-Demerdash E, Shaarawy S, El-Merzabani M and Ammar el-SM: Sensitization of TRAILinduced apoptosis in human hepatocellular carcinoma HepG2 cells by phytochemicals. Life Sci 92: 555-561, 2013.

8. Farabegoli F, Papi A, Bartolini G, Ostan R and Orlandi M: (-)-Epigallocatechin-3- gallate downregulates Pg-P and BCRP in a tamoxifen resistant MCF-7 cell line. Phytomedicine 17: 356-362, 2010.

9. Lee SH, Nam HJ, Kang HJ, Kwon HW and Lim YC: Epigallocatechin-3-gallate attenuates head and neck cancer stem cell traits through suppression of Notch pathway. Eur J Cancer 49: 3210-3218, 2013.

10. Lecumberri E, Dupertuis YM, Miralbell R and Pichard C: Green tea polyphenol epigallocatechin-3-gallate (EGCG) as adjuvant in cancer therapy. Clin Nutr 32: 894-903, 2013.

11. Qian Y, Guan T, Huang M, et al: Neuroprotection by the soy isoflavone, genistein, via inhibition of mitochondria-dependent apoptosis pathways and reactive oxygen induced-NF- $\mathrm{KB}$ activation in a cerebral ischemia mouse model. Neurochem Int 60: 759-767, 2012.

12. Forbes-Hernández TY, Giampieri F, Gasparrini M, Mazzoni L, Quiles JL, Alvarez- Suarez JM and Battino M: The effects of bioactive compounds from plant foods on mitochondrial function: a focus on apoptotic mechanisms. Food Chem Toxicol 68: 154-182, 2014.

13. Kwak TW, Kim do H, Chung CW, Lee HM, Kim CH, Jeong YI and Kang DH: Synergistic anticancer effects of vorinostat and epigallocatechin-3-gallate against HuCC-T1 human cholangiocarcinoma cells. Evid Based Complement Alternat Med 2013: 185158,2013

14. Lee JH, Jeong YJ, Lee SW, et al: EGCG induces apoptosis in human laryngeal epidermoid carcinoma Hep2 cells via mitochondria with the release of apoptosis-inducing factor and endonuclease G. Cancer Lett 290: 68-75, 2010.

15. Sakamoto Y, Terashita N, Muraguchi T, Fukusato $T$ and Kubota S: Effects of epigallocatechin-3-gallate (EGCG) on A549 lung cancer tumor growth and angiogenesis. Biosci Biotechnol Biochem 77: 1799-1803, 2013.

16. Spagnuolo P, Rasini E, Luini A, et al: Isoflavone content and estrogenic activity of different batches of red clover (Trifolium pratense L.) extracts: an in vitro study in MCF-7 cells. Fitoterapia 94: 62-69, 2014.

17. Eskandani M, Hamishehkar H and Ezzati Nazhad Dolatabadi J: Cytotoxicity and DNA damage properties of tert-butylhydroquinone (TBHQ) food additive. Food Chem 153: 315-320, 2014.

18. Valenti D, de Bari L, Manente GA, Rossi L, Mutti L, Moro L and Vacca RA: Negative modulation of mitochondrial oxidative phosphorylation by epigallocatechin-3 gallate leads to growth arrest and apoptosis in human malignant pleural mesothelioma cells. Biochim Biophys Acta 1832: 2085-2096, 2013.

19. Tran PL, Kim SA, Choi HS, Yoon JH and Ahn SG: Epigallocatechin-3-gallate suppresses the expression of HSP70 and HSP90 and exhibits anti-tumor activity in vitro and in vivo. BMC Cancer 10: 276, 2010

20. Hwang YS, Park KK and Chung WY: Epigallocatechin-3 gallate inhibits cancer invasion by repressing functional invadopodia formation in oral squamous cell carcinoma. Eur J Pharmacol 715 : 286-295, 2013
21. Singh M, Singh R, Bhui K, Tyagi S, Mahmood Z and Shukla Y: Tea polyphenols induce apoptosis through mitochondrial pathway and by inhibiting nuclear factor- $\kappa \mathrm{B}$ and Akt activation in human cervical cancer cells. Oncol Res 19: 245-257, 2011.

22. Shan X, Li Y, Meng X, Wang P, Jiang P and Feng Q: Curcumin and (-)-epigallocatechin-3-gallate attenuate acrylamide-induced proliferation in HepG2 cells. Food Chem Toxicol 66: 194-202, 2014.

23. Ma J, Shi M, Li G, et al: Regulation of Id1 expression by epigallocatechin-3-gallate and its effect on the proliferation and apoptosis of poorly differentiated AGS gastric cancer cells. Int J Oncol 43: 1052-1058, 2013.

24. Thakur VS, Ruhul Amin AR, Paul RK, et al: p53-Dependent p21-mediated growth arrest pre-empts and protects HCT116 cells from PUMA-mediated apoptosis induced by EGCG. Cancer Lett 296: 225-232, 2010

25. Tsai CF, Hsu YW, Ting HC, Huang CF and Yen CC: The in vivo antioxidant and antifibrotic properties of green tea (Camellia sinensis, Theaceae). Food Chem 136: 1337-1344, 2013.

26. Kim HS, Quon MJ and Kim JA: New insights into the mechanisms of polyphenols beyond antioxidant properties; lessons from the green tea polyphenol, epigallocatechin 3-gallate. Redox Biol 2: 187-195, 2014.

27. Satoh M, Takemura Y, Hamada H, Sekido Y and Kubota S: EGCG induces human mesothelioma cell death by inducing reactive oxygen species and autophagy. Cancer Cell Int 13: 19, 2013.

28. Manohar M, Fatima I, Saxena R, Chandra V, Sankhwar PL and Dwivedi A: (-)-Epigallocatechin-3-gallate induces apoptosis in human endometrial adenocarcinoma cells via ROS generation and p38 MAP kinase activation. J Nutr Biochem 24: 940-947, 2013.

29. Kalaiselvi P, Rajashree K, Bharathi Priya L and Padma VV: Cytoprotective effect of epigallocatechin-3-gallate against deoxynivalenol-induced toxicity through anti-oxidative and antiinflammatory mechanisms in HT-29 cells. Food Chem Toxicol 56: 110-118, 2013.

30. Susin SA, Lorenzo HK, Zamzami N, et al: Molecular characterization of mitochondrial apoptosis-inducing factor. Nature 397: 441-446, 1999.

31. Gao Y, Li W, Jia L, Li B, Chen YC and Tu Y: Enhancement of (-)-epigallocatechin-3-gallate and theaflavin-3-3'-digallate induced apoptosis by ascorbic acid in human lung adenocarcinoma SPC-A-1 cells and esophageal carcinoma Eca-109 cells via MAPK pathways. Biochem Biophys Res Commun 438: 370-374, 2013.

32. Deindl E: Mechanistic insights into the functional role of vascular endothelial growth factor and its signalling partner brain-derived neurotrophic factor in angiogenic tube formation. Acta Physiol 211: 268-270, 2014.

33. Gorman JL, Liu ST, Slopack D, et al: Angiotensin II evokes angiogenic signals within skeletal muscle through co-ordinated effects on skeletal myocytes and endothelial cells. PLoS One 9: e85537, 2014.

34. Takala H, Saarnio J, Wiik H, Ohtonen P and Soini Y: HIF-1 $\alpha$ and VEGF are associated with disease progression in esophageal carcinoma. J Surg Res 167: 41-48, 2011.

35. Hsieh DS, Wang H, Tan SW, Huang YH, Tsai CY, Yeh MK and Wu CJ: The treatment of bladder cancer in a mouse model by epigallocatechin-3-gallate-gold nanoparticles. Biomaterials 32: 7633-7640, 2011

36. Shimizu M, Shirakami Y, Sakai H, et al: (-)-Epigallocatechin gallate inhibits growth and activation of the VEGF/VEGFR axis in human colorectal cancer cells. Chem Biol Interact 185: 247-252, 2010.

37. Valenti D, De Rasmo D, Signorile A, et al: Epigallocatechin-3gallate prevents oxidative phosphorylation deficit and promotes mitochondrial biogenesis in human cells from subject with Down's syndrome. Biochim Biophys Acta 1832: 542-552, 2013.

38. Galati G, Lin A, Sultan AM and O'Brien PJ: Cellular and in vivo hepatotoxicity caused by green tea phenolic acids and catechins. Free Radic Biol Med 40: 570-580, 2006.

39. Min NY, Kim JH, Choi JH, et al: Selective death of cancer cells by preferential induction of reactive oxygen species in response to (-)-epigallocatechin-3-gallate. Biochem Biophys Res Commun 421: 91-97, 2012. 